\title{
4Pi-microscopy of the Golgi apparatus in live mammalian cells
}

\author{
Alexander Egner, ${ }^{\mathrm{a}}$ Sophie Verrier, ${ }^{\mathrm{b}}$ Alexander Goroshkov, ${ }^{\mathrm{a}}$ \\ Hans-Dieter Söling, ${ }^{\mathrm{b}}$ and Stefan W. Hell ${ }^{\mathrm{a}, *}$ \\ ${ }^{a}$ Department of NanoBiophotonics, Max-Planck-Institute for Biophysical Chemistry, High Resolution Optical Microscopy Group, \\ 37077 Göttingen, Germany \\ b Department of Neurobiology, Max-Planck-Institute for Biophysical Chemistry, 37077 Göttingen, Germany
}

Received 9 September 2003, and in revised form 3 October 2003

\begin{abstract}
We report the applicability of 4Pi-microscopy to live mammalian cells. Controlled interference of the counterpropagating wavefronts is possible despite the slight variations in cellular refractive index. Superresolved 3D-fluorescence imaging is exemplified with the first representation of the Golgi apparatus in a live cell at $\sim 100 \mathrm{~nm}$ resolution.
\end{abstract}

(C) 2003 Elsevier Inc. All rights reserved.

\section{Introduction}

Catalyzed by the advent of specific exogenous and endogenous markers, such as the green fluorescent protein (GFP) and its mutants, far-field fluorescence microscopy techniques have evolved into powerful tools in the life sciences. By facilitating noninvasive three-dimensional (3D-) imaging of the interior of live cells, confocal and multiphoton fluorescence microscopes have further added to this power (Pawley, 1995). However, continual progress of far-field microscopy is hampered by the limited spatial resolution which is at its best $\sim 180$ and $500 \mathrm{~nm}$ in the lateral and axial direction, respectively (Pawley, 1995). These limits inevitably hinder the detailed observation of cellular compartments in live cells.

The axial resolution can be improved by 3-7 times through the coherent addition of the focused wavefronts of two opposing lenses, as pursued in 4Pi-microscopy (Hell and Stelzer, 1992) and the related $\mathrm{I}^{5} \mathrm{M}$ technique (Gustafsson et al., 1995, 1999). The aperture enlargement is particularly powerful in connection with multiphoton excitation, because the nonlinear dependence of the fluorescence on the local intensity, as well as the disparity between the excitation and fluorescence

\footnotetext{
${ }^{*}$ Corresponding author. Fax: +49-551-201-1085.

E-mail address: shell@gwdg.de (S.W. Hell).
}

wavelengths reduces the effect of interference sidelobes (Hell and Stelzer, 1992). In fact, the avoidance of ambiguities resulting from the sidelobes is the actual physical challenge in exploiting interference for axial resolution improvement. Therefore, confocalization is routinely added to 4Pi-imaging as a further precaution against sidelobes. The result is that multiphoton 4Piconfocal microscopy has delivered sharper images in conjunction with all mounting media, and most notably with aqueous solutions (Bahlmann et al., 2001; Egner et al., 2002). The latter is of course a prerequisite for imaging live cells (Egner et al., 2002).

Recently, a multifocal version of a multiphoton excitation microscope of type A has been developed that enabled fast imaging through swiftly scanning 16-64 pairs of interfering excitation spots in parallel (Egner et al., 2002). Briefly referred to as the MMM-4Pi, this microscope has been used for the 3D-imaging of live budding yeast cells (Saccharomyces cerevisiae). In combination with nonlinear image restoration, the MMM4Pi provided an unprecedented $\sim 100 \mathrm{~nm} 3 \mathrm{D}$-resolution of the yeast mitochondrial compartment (Egner et al., 2002). However, it still remained unclear whether it would also be applicable to live mammalian cells. Relying on opposing high aperture lenses, 4Pi-microscopy usually requires the mounting of the cells between two cover slips, which raises questions about prolonged cell viability. In addition, it has been speculated that cell-induced refractive index changes would spoil the 
controlled production of constructive interference (Lindek et al., 1994). This stems from the fact that the structure of the focal spot, i.e., the effective pointspread-function (E-PSF) of the 4Pi-microscope strongly depends on the relative phase of the interfering wavefronts. Unfortunately, any local variation in the sample's refractive index affects the phase difference and hence alters the structure of the E-PSF (Blanca et al., 2002; Egner et al., 1998). While it has been shown that for glycerol-mounted mammalian cells the refractive index variations are comparatively small (Egner et al., 1998; Hell et al., 1997; Nagorni and Hell, 1998), these results cannot be extrapolated to live cells. The reason is that, compared to the refractive index of 1.46 of glycerol, the corresponding value of water (1.33) is further away from the 1.48 and 1.54 values of lipid membranes and proteins, respectively. Therefore, the complex distribution of refractive indices in a live cell has questioned the general applicability of 4Pi-microscopy.

Here we apply the MMM-4Pi system to the imaging of two protein distributions of a live Vero cell, that are reportedly constituents of the Golgi apparatus (Keenan, 1998): UDP-galactosyl: glycoprotein, $1,4-\beta$-galactosyl transferase (GalT) and $3^{\prime}$-phosphoadenylyl-sulfate: uronyl-2-O-sulfotransferase (2-OST). In doing so, we provide initial evidence for the practicality of 4Pi-microscopy in live mammalian cells. Moreover, our application yields the first images of a live Golgi apparatus at a 3D-resolution of $\sim 100 \mathrm{~nm}$.

\section{Methods}

\subsection{DNA constructs}

Escherichia coli strain XL1-Blue was used to amplify plasmid DNA. All constructs were verified by DNA sequencing. For transfection of Vero cells, endotoxin-free plasmid DNA was prepared from E. coli strain XL1-Blue using the Endotoxin-free Plasmid Kit (Qiagen). A cDNA coding for amino acids 1-60 of GalT was cloned into the pEGFP-N1 vector (Clontech). The cDNA was kindly provided by Dr. J. Lippincott-Schwartz and Dr. N. Altan-Bonnet (NIH, Bethesda, MD) (Ward et al., 2001). It had previously been shown that this truncated GalT which contains 17 amino acids of the lumenal domain, the transmembrane domain, and the $\mathrm{N}$-terminal cytoplasmic tail, shows the same Golgi distribution as wild-type GalT (Cole et al., 1996). A cDNA encoding the full-length 2-OST was amplified by RT-PCR (Qiagen kit) from mouse mRNA, using as forward primer $5^{\prime}$-AT TCAGGAATTCTGCCACCATGGGGCTCCTCAGG ATTATG- $3^{\prime}$ and as reverse primer $5^{\prime}$-ATTCAGGGA TCCTCGTTCGACTTAGGGTAAATC- $3^{\prime}$. The PCR product was ligated into pEGFP-N1 (Clontech) using EcoRI/BamHI restriction sites.

\subsection{Cell culture and transfection}

Vero cells (African green monkey kidney cells; ECACC 84113001) were grown in DMEM with $2 \mathrm{mM}$ glutamine, $10 \%$ heat-inactivated fetal calf serum, 100 $\mathrm{U} / \mathrm{ml}$ penicillin, and $0.1 \mathrm{mg} / \mathrm{ml}$ streptomycin at $10 \% \mathrm{CO}_{2}$ and $37^{\circ} \mathrm{C}$. For DNA transfection, Vero cells were split in $100-\mathrm{mm}$ dishes two days before transfection, so that the cells were confluent on the day of transfection. Cells were then trypsinized, washed with PBS, and the pellet was resuspended in the DNA solution containing $10 \mu \mathrm{g}$ of DNA in $400 \mu \mathrm{l}$ cytomix $(120 \mathrm{mM} \mathrm{KCl}, 10 \mathrm{mM}$ $\mathrm{KH}_{2} \mathrm{PO}_{4}, 10 \mathrm{mM} \mathrm{K} \mathrm{HPO}_{4}, 2 \mathrm{mM}$ EGTA, $5 \mathrm{mM} \mathrm{MgCl}$, $25 \mathrm{mM}$ Hepes, $0.15 \mathrm{mM} \mathrm{CaCl}_{2}, 5 \mathrm{mM} \mathrm{GSH}$, and $2 \mathrm{mM}$ ATP, pH 7.4). Cells were immediately electroporated (pulse $0.7 \mathrm{kV}, 50 \mu \mathrm{F}, 1-2 \mathrm{~ms}$ ). Twenty four hours before image acquisition, transfected Vero cells were grown in a self-constructed closed flow chamber that accommodated the two cover slips at $\sim 175 \mu \mathrm{m}$ distance. The addition of an air/ $/ \mathrm{CO}_{2}$ mix to the culture medium extended the viability of the cells to over $48 \mathrm{~h}$. Division and propagation were maintained. All experiments were carried out at an elevated room temperature of $32^{\circ} \mathrm{C}$.

\subsection{Multifocal multiphoton 4Pi microscopy}

Two-photon excitation of EGFP was performed at a wavelength of $870 \mathrm{~nm}$, with an array of $164 \mathrm{Pi}$-foci of $\sim 1.5 \mathrm{~mW}$ average power each. The excitation light originated from a mode-locked Ti-Sapphire laser (Mai Tai, Spectra Physics) and the fluorescence was imaged onto a back-thinned cooled CCD-camera (Egner et al., 2002). The raw 4Pi-data were nonlinearly deconvolved with a measured E-PSF as described in the literature (Nagorni and Hell, 2001). The deconvolution automatically removed the sidelobes in the resulting image. The linear phase shift induced by the residual difference between the refractive index of the water immersion liquid and the mounting medium (culture growth medium DMEM from Invitrogen, plus Dextran MW 39,400; $3.4 \%$ of total weight, from Sigma) was actively compensated (Egner et al., 1998). Typical stacks comprised $100 x y$-images that were axially separated by $80 \mathrm{~nm}$. Hence the typical recording volume within the cell was $16 \times 16 \times 8 \mu^{3}$. The recording time per image was $2 \mathrm{~s}$ ( $1 \mathrm{~s}$ exposure and $1 \mathrm{~s}$ camera readout), which is not much slower than standard beam-scanning confocal microscopy. The recording time can be easily cut down by half by using a technically more advanced and faster CCDcamera that is already commercially available.

\subsection{Preparation of ultrathin fluorescent layers on cover slips}

Cover slips were firmly coated with the fluorescent dye Oregon Green (Molecular Probes, OR, USA) 
through a standard silanization procedure (de Leon and Biewer, 2000). The coating resulted in an ultrathin $(<3 \mathrm{~nm})$ fluorescent layer whose axial scanning results in a $z$-response function that discloses the axial structure of the effective point-spread-function of the microscope.

\section{Results}

\subsection{Optical conditions and cell-induced phase alterations}

To explore whether the optical conditions for 4Piimaging can be fulfilled, we grew Vero cells on cover slips coated with an ultrathin layer of Oregon Green, a two-photon excitable fluorophore emitting around $525 \mathrm{~nm}$. The cells were covered with a similar coverslip so that they were essentially sandwiched between two ultrathin fluorescent layers. The aqueous mounting medium featured a refractive index of 1.34 .

Fig. 1 shows an $x z$-section of a typical 3D-data stack containing a part of the nucleus. The $x z$-section through the ultrathin layers on the cover slips and the resulting $z$-profile disclose the quality of the interference between the two wavefronts. Three profiles are extracted from three different sites in the nuclear region, in which the local variations in refractive index are strongly pronounced: completely outside the nucleus, at the nuclear periphery, and straight through the nucleus. Although slight changes become apparent, the phase does not notably change with the axial translation. This can be inferred from the comparison between the profiles in the same line, pertinent to the different cover slips.

Comparison of the profiles from the same coverslip reveals that the presence of the nucleus introduces aberrations leading to elevated sidelobes, but again the changes in refractive index are not pronounced enough to distort the E-PSF. In the perinuclear region, the sidelobe height is lower than the critical value of $50 \%$ of the central peak, but if the nucleus is directly involved, the lobes are slightly elevated. This indicates that two-photon excitation and confocalization are currently critical to the applicability in live cells. The upper profile also shows that the refractive index challenges are relaxed when largely omitting the nucleus-cytosol interface. In brief, the data in Fig. 1 confirm that the optical conditions are fulfilled for the 3D-imaging of the Golgi apparatus under live cell conditions.

\subsection{High-resolution imaging of the Golgi enzymes GalT and 2-OST}

In addition to important metabolic functions, proteins residing in the Golgi carry out functions associated with post-translational modifications of secretory proteins, such as glycosylation, sulfation and proteolytic processing, membrane transport, recycling of endoplasmic reticulum proteins, and maintenance of the structural organization of the organelle itself. Isolation of enriched Golgi membrane fractions led to the discovery that the glycosyltransferase responsible for the transfer of galactosyl moieties in $\beta-1,4$ linkage into glycoproteins (GalT), namely $\beta 1$,4-galactosyltransferase $(\beta 1,4 \mathrm{GalT})$, was highly enriched in Golgi membranes (Schachter et al., 1970). This finding provided a marker enzyme for the Golgi compartment during cell fractionation and has remained the marker of choice until today. Immunocytochemical studies indicated that GalT was confined mainly to the trans-Golgi cisternae (Roth and Berger, 1982). Therefore, we imaged the 3D-distribution of a fusion protein between EGFP and a truncated form of GalT. It has previously been shown that the localization of GalT is determined by its transmembrane domain and that localization of the truncated GalT used here is the same as that of wild-type GalT (Cole et al., 1996).

To determine to what extent movements of the live Golgi apparatus affect 4Pi-imaging we recorded a series of three subsequent 3D-stacks of the GalT-EGFP distribution in a live Vero cell. Fig. 2 shows three consecutive $x z$-recordings of an arbitrary site of the Golgi with a time difference of $210 \mathrm{~s}$, extracted from consecutive recordings of 4Pi-data stacks (Fig. 2, bottom). The data reveals that at the employed $32{ }^{\circ} \mathrm{C}$ this time difference is long enough to witness slight morphological changes in the Golgi, but these changes are much too small to affect 4Pi-imaging. This can be inferred from the fact that the lobe-induced 'ghosts' in the raw data exhibit identical structures on either side. This is not surprising, since at given recording times, it takes only $20 \mathrm{~s}$ to scan a point of the object through the volume of the 4Pi-PSF. Hence in spite of the slight morphological changes occurring in the live cell, the data can be deconvolved for achieving a $\sim 100 \mathrm{~nm}$ 3D-resolution (Fig. 2, top). In addition, the 3-fold recording of the Golgi apparatus did not witness a significant reduction in image brightness. Therefore, Fig. 2 also shows that photobleaching of EGFP was not an issue in this work.

The inset in the upper left-hand corner of Fig. 3 displays a conventional overview $X Y$-image of the EGFP-labeled Golgi apparatus located close to the Hoechst-counterstained nucleus. The central panel displays the corresponding $3 \mathrm{D}$ surface-rendered image at $100 \mathrm{~nm}$ resolution. The 3D-image clearly reveals ribbons, fractioned stacks, as well as smaller tubular, and vesicular subcompartments. Furthermore, protrusions and invaginations in the $200 \mathrm{~nm}$ range that are hardly discernible in confocal image can be noticed. The inset in the upper right-hand corner is a composite of several 

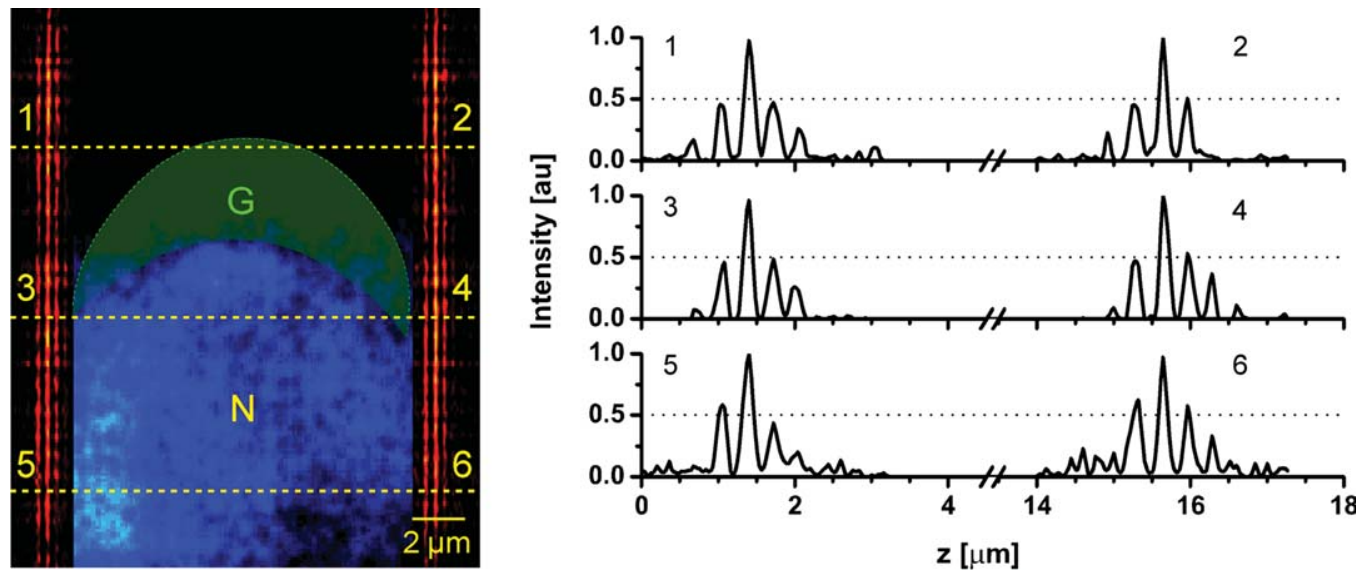

Fig. 1. (left) $X Z$-section recorded at the periphery of the nucleus of a live Vero cell sandwiched between two cover slips each thinly coated with a fluorescence layer. The nucleus is highlighted through Hoechst counterstaining $(\mathrm{N})$ and outlined; the region where the Golgi resides is briefly sketched (G). The cell is significantly larger than the whole $x z$-section. The three vertical lines on either side (orange) are due to the interference between the counterpropagating focused field, representing the main maximum of the 4Pi-PSF and the two axially offset sidelobes. (right) displays enlarged intensity profiles of the axial responses of the microscope to the thin fluorescent layers, revealing the height of the lobes and the relative phase in greater detail. Outside $(1 \& 2)$ and at the edge $(3 \& 4)$ of the nucleus, the primary lobes are below the critical value of $50 \%$. Focusing through the nucleus ( 5 \& 6) slightly increases the lobe height and induces a noticeable but comparatively small phase shift. The generally maintained parallelism of the orange lines in (A) indicates that the cell does not gravely distort the interference between the wavefronts.
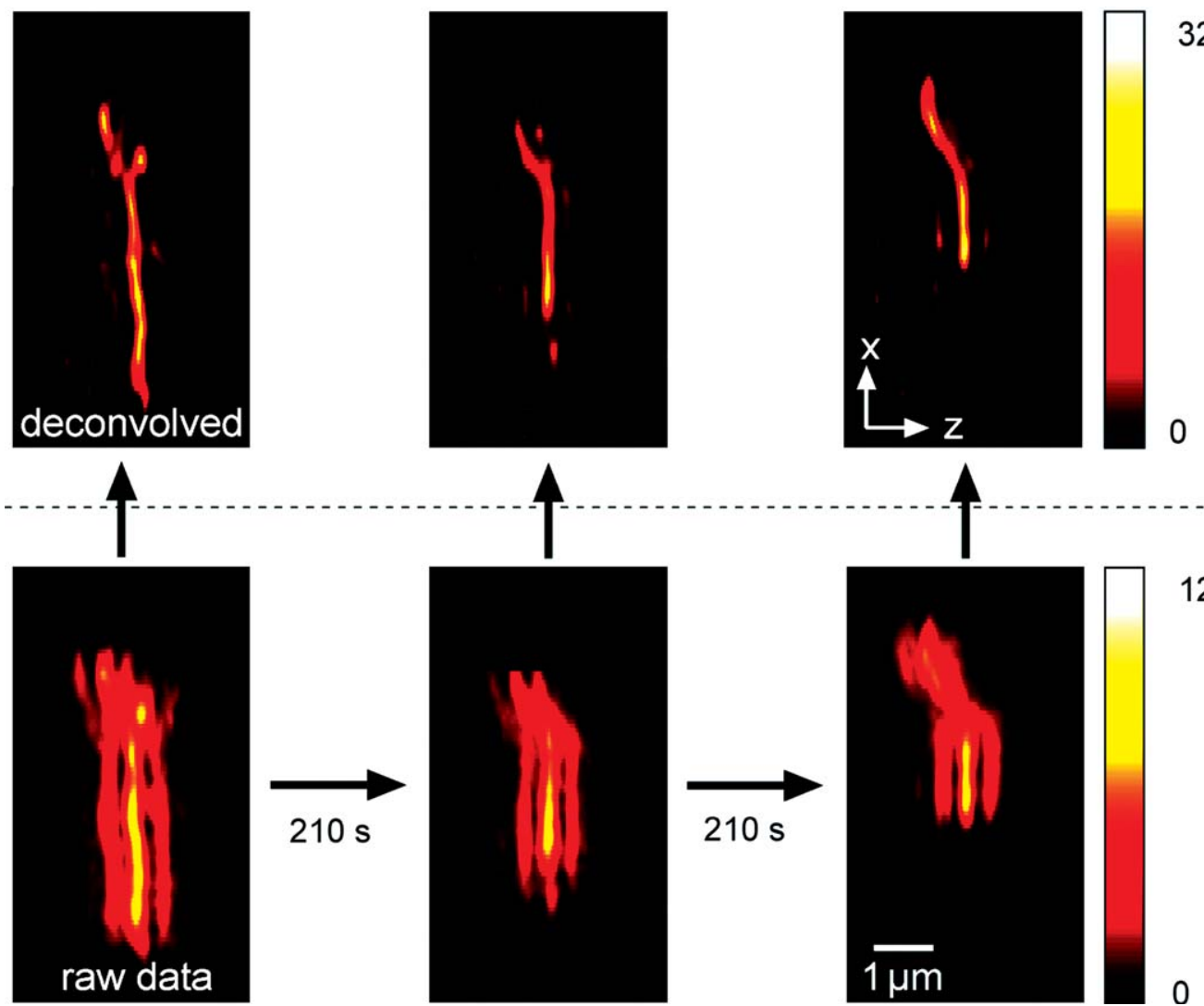

Fig. 2. Raw (bottom) and deconvolved (top) 4Pi-data from the same $X Z$-section $(6.8 \times 3.7 \mu \mathrm{m})$ of three successively recorded 3D-stacks of the GalTEGFP-labeled Golgi apparatus of a live Vero cell. The time difference between the recordings was $210 \mathrm{~s}$. The parallel 'stripes' on either side of the structure are due to the lobes inherent to the 4Pi-PSF (bottom); they are removed by mathematical filtering (top). The duration of $210 \mathrm{~s}$ was long enough to witness morphological changes within the live organelle, but these changes are too slow to affect the high-resolution 4Pi-recording of live cells. This can be inferred from the fact that the lobe-induced stripes on either side of the structure are very similar (bottom), thus enabling devonvolution. Besides, featuring similar brightness the consecutive recordings indicate high bleaching stability of the utilized EGFP marker. 


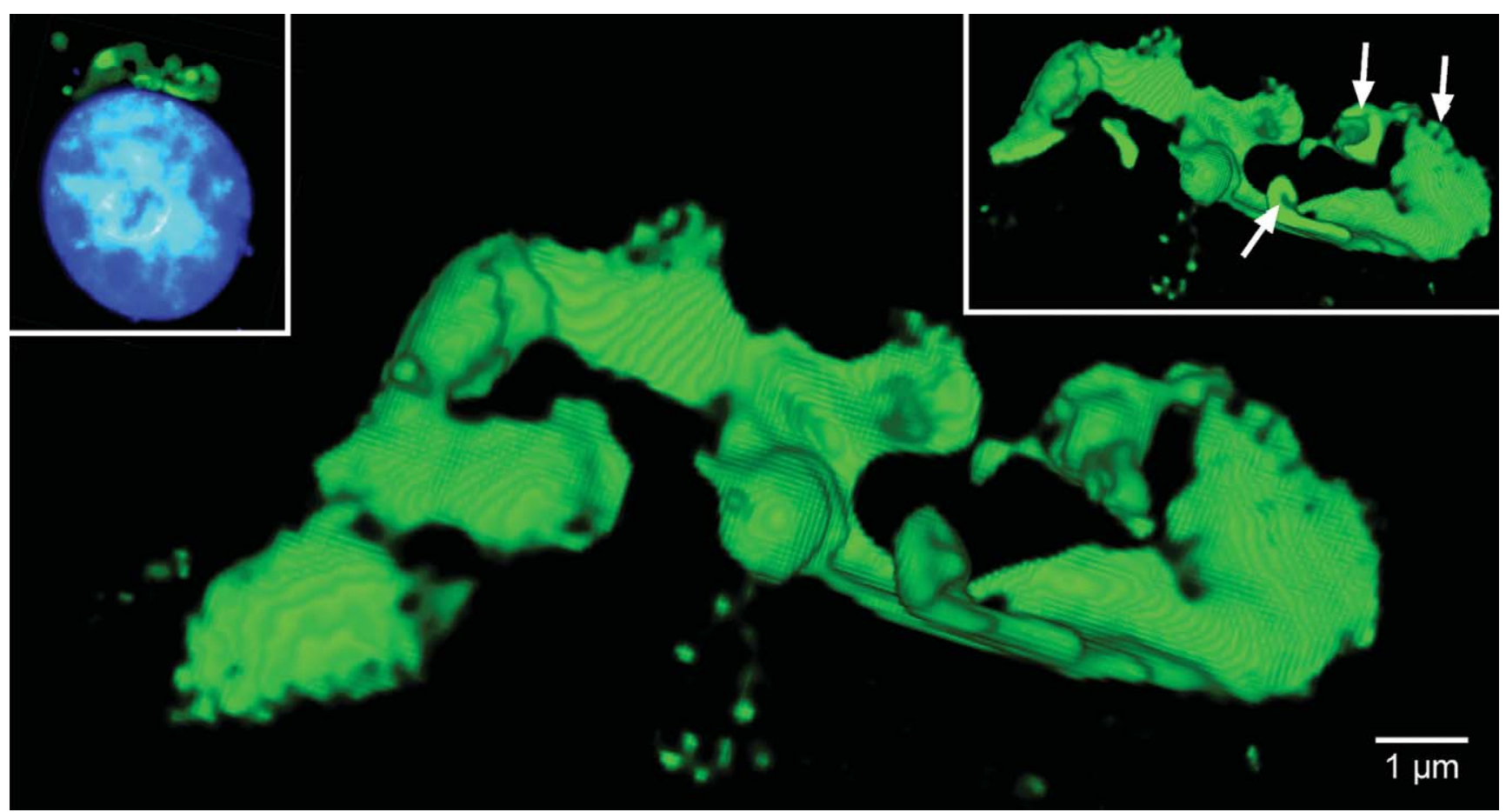

Fig. 3. Golgi apparatus as obtained through the 4Pi-confocal imaging of GalT-EGFP in a live Vero cell at $\sim 100 \mathrm{~nm}$ spatial resolution. This protein is primarily resident in the medial and trans part of the Golgi-apparatus. The image has been obtained through voltex-rendering of a stack of $10.5 \times 14.8 \times 6.4 \mu^{3}$ in size. Note the convoluted structure of the Golgi apparatus featuring ribbons and fractionated stacks, as well as smaller tubular and vesicular subcompartments. The striped features inside the image are generated by the volume rendering algorithm and therefore not present in the data. (Left-hand inset) Epifluorescence image to correlate the Golgi with the nucleus. The Hoechst-staining of the nucleus indicates that the cell was in interphase. (Upper right-hand inset) Composite picture of the same cell, showing membrane protrusions and invaginations (arrows). For this example, only sections from the lower half of the image stack were used. A movie of the 3D image is provided online (Movie 1).
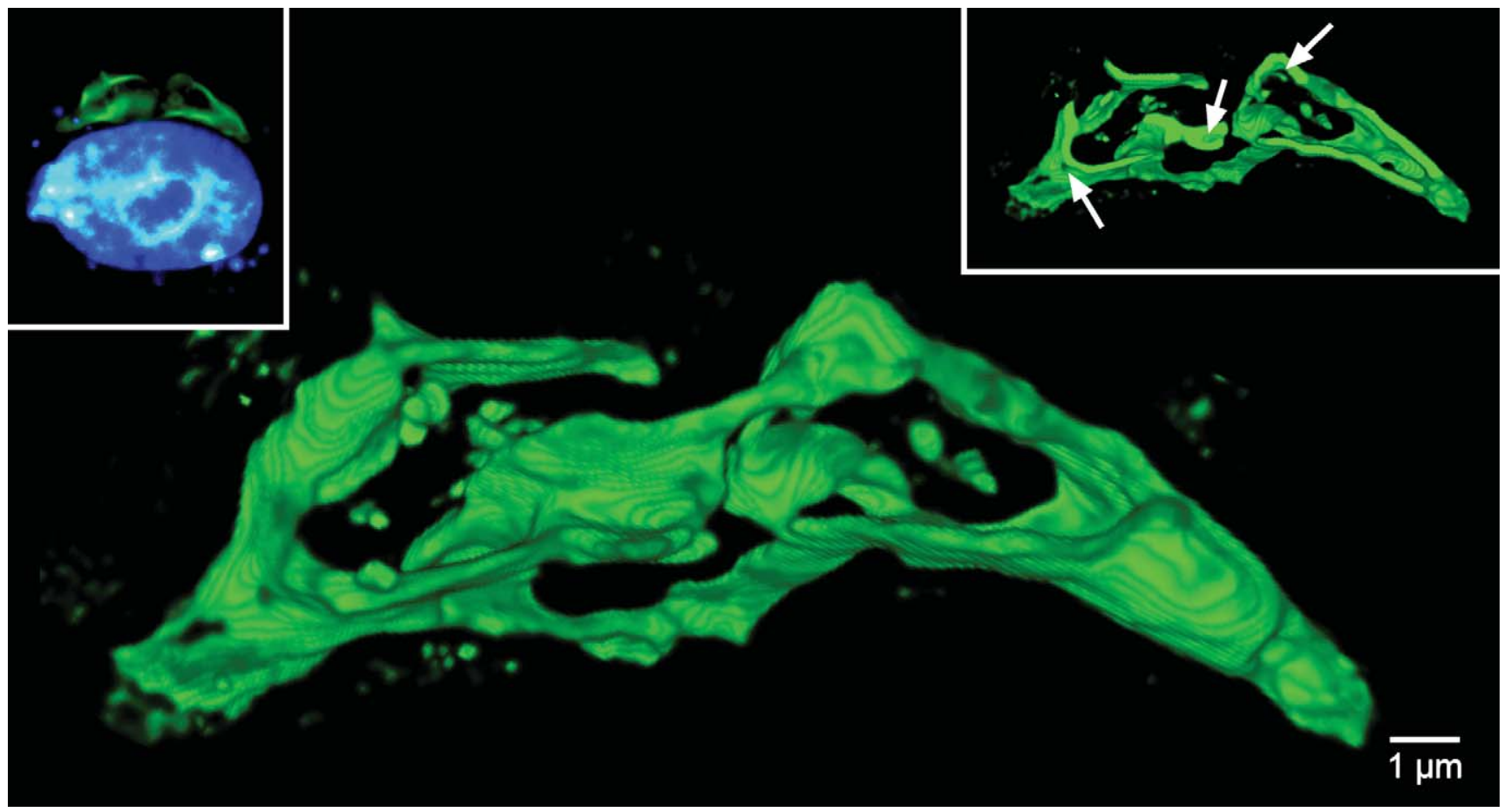

Fig. 4. Golgi apparatus of a live Vero cell as represented by the enzyme-2-OST-EGFP. The image size is $19.0 \times 11.7 \times 8.0 \mu \mathrm{m}^{3}$; the data representation is the same as in Fig. 3. This protein is reportedly present in the cis and medial Golgi apparatus. A movie of this 3D-representation is provided online (Movie 2). 
serial sections showing the 3D configuration of the Golgi apparatus, illustrating the rolling arrangement of the connected Golgi-stacks. A 3D-movie of this 3Drepresentation is provided in the supplementary information.

Fig. 4 shows a typical distribution of 2-OST-EGFP in interphase cells. It has been argued that this Golgi enzyme exists mainly in the cis-Golgi cisternae (Pinhal et al., 2001). However, for most enzymes involved in proteoglycan synthesis, the exact localization within the Golgi has remained unclear. The 4Pi-3D-image obtained for the distribution of 2-OST also displays a convoluted structure of the Golgi apparatus, similar to that observed for GalT. However, a discrimination of intraGolgi localization of 2-OST and GalT will be possible by applying two color MMM-4Pi-microscopy which is presently under development.

The cells remained viable even after prolonged mounting in the self-made chamber for several days. The comparatively large space of $175 \mu \mathrm{m}$ between the two coverslips and the addition of a suitable air/ $\mathrm{CO}_{2}$ mix is adequate to maintain division and propagation without apparent degradation.

\section{Discussion and conclusion}

The series of images obtained for the distribution of the two Golgi enzymes constitute what is, to our knowledge, the most detailed visualization of an organelle in a live mammalian cell. We found that the phase changes induced by refractive index variations do not preclude multiphoton 4Pi-confocal imaging. Even in the closest proximity to the nucleus, the phase changes are weak enough not to fundamentally alter the phase difference between the focused illumination wavefronts. We also found that at the utilized temperature of $32^{\circ} \mathrm{C}$ movements within the cell are sufficiently reduced. Therefore, they do not affect 4Pi-imaging at the present image acquisition speed. These results clearly demonstrate the applicability of 4Pi-microscopy to live mammalian cells.

Still, it is important to bear in mind that the structure of the 4Pi-PSF was greatly stabilized by the nonlinear excitation mode and the confocal suppression of the axially offset sidelobes. Therefore, abandoning multiphoton excitation or confocalization would probably compromise the practicability of this interference based technique in live cells.

Future technical improvements will encompass the implementation of faster CCD-cameras and the extension to multicolor detection. We anticipate that multicolor imaging should enable the superior localization of different proteins paired with superior morphological information. These advantages may eventually allow the tackling of several of the currently debated questions about the Golgi apparatus (Marsh and Howell, 2002). Furthermore, our results indicate that 4Pi-microscopy should be extendable to other organelles in live cells, as well as to the distribution of other proteins in substructures of a given compartment.

\section{Acknowledgments}

We thank Katrin Barnewitz for providing us with the 2-OST-EGFP and Andreas Schönle for help with his image analysis software IMSPECTOR. We also thank Rainer Pepperkok and Philippe Bastiaens (both EMBL, Heidelberg) for motivating discussions.

\section{References}

Bahlmann, K. et al., 2001. 4Pi-confocal microscopy of live cells. Ultramicroscopy 87, 155-164.

Blanca, C.M. et al., 2002. Determination of the unknown phase difference in $4 \mathrm{Pi}$-confocal microscopy through the image intensity. Opt. Commun. 206, 281-285.

Cole, N.B. et al., 1996. Diffusional mobility of Golgi proteins in membranes of living cells. Science 273, 797-801.

de Leon, L., Biewer, M.C., 2000. Preparation of self-assembled monolayers with specific intermolecular interactions. Tetrahedron Lett. 41, 3527-3530.

Egner, A. et al., 2002. Fast 100-nm resolution 3D-microscope reveals structural plasticity of mitochondria in live yeast. Proc. Natl. Acad. Sci. USA 99, 3370-3375.

Egner, A. et al., 1998. Refractive index mismatch induced intensity and phase variations in fluorescence confocal, multiphoton and 4Pimicroscopy. Opt. Commun. 153, 211-217.

Gustafsson, M.G.L. et al., 1995. Sevenfold improvement of axial resolution in 3D widefield microscopy using two objective lenses. Proc. SPIE 2412, 147-156.

Gustafsson, M.G.L. et al., 1999. I5M: 3D widefield light microscopy with better than $100 \mathrm{~nm}$ axial resolution. J. Microsc. 195, 10-16.

Hell, S.W. et al., 1997. Far-field fluorescence microscopy with threedimensional resolution in the $100 \mathrm{~nm}$ range. J. Microsc. 185, 1-5.

Hell, S.W., Stelzer, E.H.K., 1992. Fundamental improvement of resolution with a $4 \mathrm{Pi}$-confocal fluorescence microscope using twophoton excitation. Opt. Commun. 93, 277-282.

Keenan, T.W., 1998. Biochemistry of the Golgi apparatus. Histochem. Cell Biol. 109, 505-516.

Lindek, S. et al., 1994. Theta microscopy allows phase regulation in 4Pi(A)-confocal two-photon fluorescence microscopy. Optik 98, 15-20.

Marsh, B.J., Howell, K.E., 2002. The mammalian Golgi complex debates. Nat. Rev./Mol. Cell Biol. 3, 789-795.

Nagorni, M., Hell, S.W., 1998. 4Pi-confocal microscopy provides three-dimensional images of the microtubule network with 100 to150-nm resolution. J. Struct. Biol. 123, 236-247.

Nagorni, M., Hell, S.W., 2001. Coherent use of opposing lenses for axial resolution increase in fluorescence microscopy. II. Power and limitation of nonlinear image restoration. J. Opt. Soc. Am. A. 18, 49-54.

Pawley, J., 1995. Handbook of Biological Confocal Microscopy. Plenum Press, New York.

Pinhal, A.S. et al., 2001. Enzyme interactions in heparan sulfate biosynthesis: uronosyl 5-epimerase and 2-O-sulfotransfer- 
ase interaction in vivo. Proc. Natl. Acad. Sci. USA 98, 12984 12989.

Roth, J., Berger, E.G., 1982. Immunocytochemical localization of galactosyltransferase in HeLa cells: codistribution with thiamine pyrophosphatase in trans-Golgi cisternae. J. Cell Biol. 93, 223-229.
Schachter, H. et al., 1970. Intracellular localization of liver sugar nucleotide glycoprotein glycosyltransferases in a golgi-rich fraction. J. Biol. Chem. 245, 1090-1100.

Ward, T.H. et al., 2001. Maintenance of Golgi structure and function depends on the integrity of ER export. J. Cell Biol. 155, 557-570. 